\title{
Corona Radiata - its Image after Ovulation Stimulation
}

\author{
B. Jodlowska-Jedrych, W. Matysiak \\ Department of Histology and Embryology, Medical University of Lublin, \\ e-mail: b.jedrych@gmail.com ,11 Street Radziwillowska, 20-080 Lublin, Poland
}

The number of granule cells in human pre-ovulatory follicle is approximately $50 \times 10^{6}$. The population of such cells is varied, both in morphological and functional aspects. Among granule cells there exist the following junctions: gap junctions, nexus [1]. Such junctions are the way of transmitting extracellular and intracellular signals between granule cells, and also between oocyte surface, or zona pellucida to be more precise, and adjacent cells. The first investigated function of granule cells in primary ovarian follicles of the rat is the production of 17 beta-estradiol. The second function is supporting the growth of oocyte by surrounding it in the follicle [2]. The studies conducted on rats and mice by Epigg et al. imply bidirectional, paracrine effects of the oocyte and granule cells on mutual development and functioning [3-4]. The studies conducted on oocyte cell cultures and granule cells of female rats ovarian follicles have showed that oocytes are an indispensable element responsible for the mechanism of estrogen and FSH effects on granule cells [5].

The aim of the study was ultrastructural evaluation of corona radiata granule cells as a result of stimulated ovulation. In the experiment there were used mature female white rats of Wistar strain with body mass of $250-300$ grams, aged $2,5-3$ months.

The rats received menopausal gonadotrophin (HMG) in single intramuscular injections (08:00 $\mathrm{hrs}$ ), then, after 10 hours (18:00 hrs), they were given a preparation of chorionic gonadotrophin hCG once. The drugs were administered on 3rd day of the estrous cycle, in the volume of $0,1 \mathrm{ml}$, by turns in the muscles of the left and right thighs.

The following subgroups of animals were identified:

A - females of this subgroup received single intramuscular injections of HMG preparation in the dose of 1,9 m.u., and then, after 10 hours they were given a single injection of hCG in the dose of 7,5 m.u.

B - animals of this group were administered HMG once, in the dose of 3,6 m.u., and at the same time as in group A, hCG in the dose of 15 m.u.

Group $\mathrm{C}$ consisted of females which received single injections of HMG preparation in the dose of 5,6 m.u. and after 10 hours hCG in the dose of 22,5 m.u.

Each subgroup consisted of 5 females. Females of this group were decapitated 14 hours after administration of the last dose of hCG. Ovaries were taken for examination. Ultrastructural evaluation of the examined material was conducted using transmission electron microscope Zeiss EM 900.

A part of corona radiata cells were tightly adjacent to one another. Among others there was finegrained, electron light substance with the structure of follicular liquid. The majority of granule cells creating the described structure had nuclei with regular contours. In the other cells the contours were irregular, with the presence of depressions and single invaginations. The cytoplasm was dominated by dilated canals of rough endoplasmic reticulum. Their interior was filled with fine-grained, electron light content, moreover, there were numerous ribosomes (Fig. 1). Greater ultrastructural changes in corona radiate cells were observed when the doses 
of administered hormones increased. In the conditions of stimulated ovulation corona radiata granule cells, perhaps as a consequence of functional damage, lost the ability of connecting proteins synthesis, which resulted in incomplete corona radiata around the oocytes. Ultrastructural changes in corona radiata granule cells, in the conditions of the described experiment, may prove the existence of a functional bond between the two structures.

\section{References}

[1] J. E. Gittens et all., Am. J. Physiol. Cell. Physiol. 284 (2003) 880.

[2] R. Buccione et all., Biol. Reprod. 43 (1990) 543.

[3] J. J. Eppig et all., Biol. Reprod. 56 (1997) 976.

[4] J. J. Eppig and K. Wigglesworth, Biol. Reprod. 63 (2000) 1014.

[5] F. Otsuka et all., Endocrinology. 146, (8) (2005) 3362.

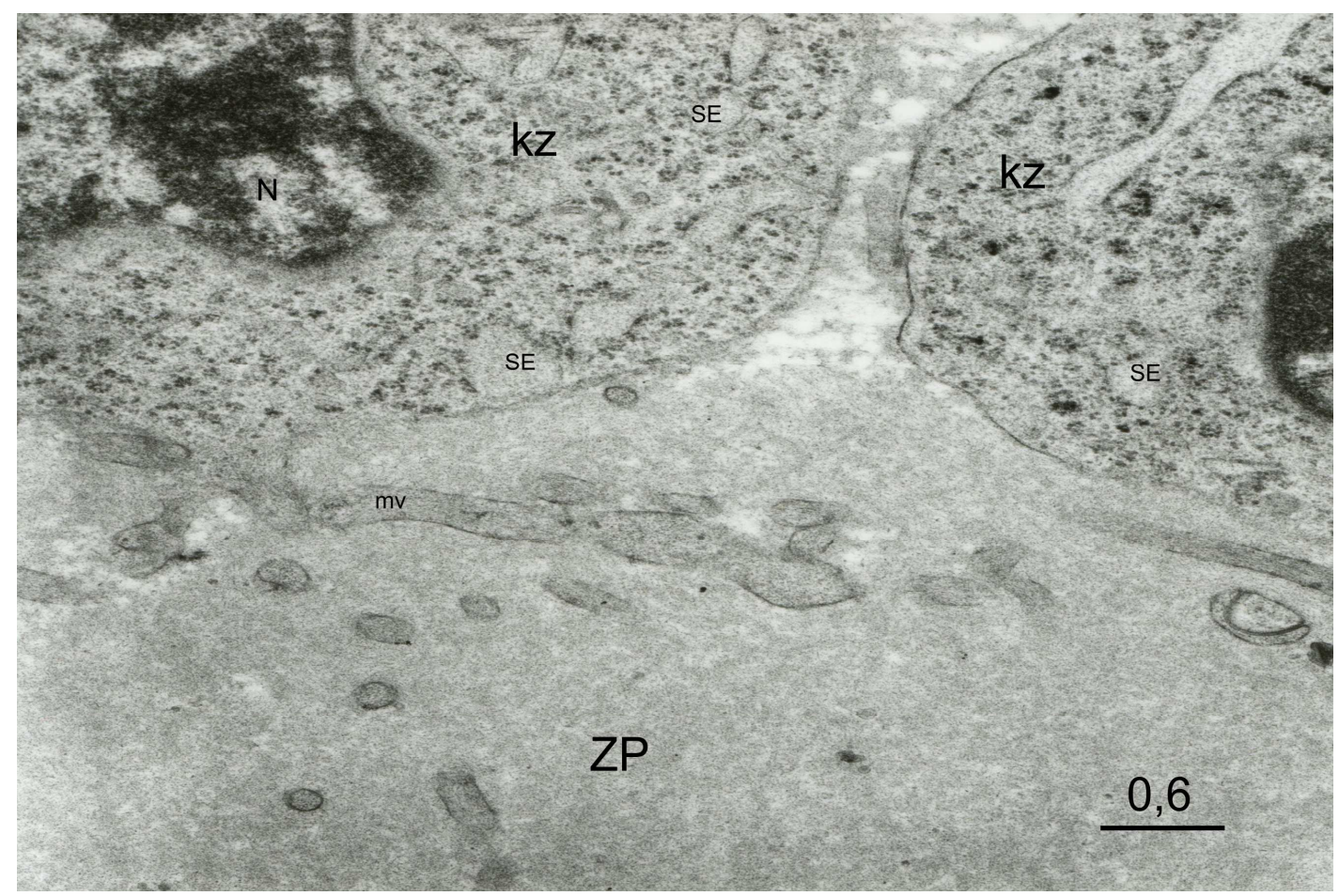

Fig. 1. Corona radiata. Group C. $\mathrm{Zp}$ - zona pellucida, $\mathrm{kz}$ - granule cells, $\mathrm{SE}$ - rough endoplasmic reticulum. Scale bar $=\mu \mathrm{m}$ 